\title{
Корреляция оптических и магнитных свойств кристаллов $\mathrm{Bi}_{2} \mathrm{Te}_{3}-\mathrm{Sb}_{2} \mathrm{Te}_{3}$
}

\author{
(C) Н.П. Степанов ${ }^{1}$, А.К. Гильфранов ${ }^{2}$, Е.Н. Трубицына ${ }^{2}$ \\ 1 Забайкальский государственный университет, \\ 672039 Чита, Россия \\ 2 Забайкальский институт предпринимательства, \\ 672039 Чита, Россия \\ E-mail: NP-Stepanov@mail.ru \\ Поступила в Редакцию 7 февраля 2019 г. \\ В окончательной редакции 10 февраля 2019 г. \\ Принята к публикации 14 февраля 2019 г.
}

\begin{abstract}
В ходе исследования кристаллов $\mathrm{Bi}_{2} \mathrm{Te}_{3}-\mathrm{Sb}_{2} \mathrm{Te}_{3}$ изучались их магнитные и оптические свойства в зависимости от соотношений компонент $\mathrm{Bi}_{2} \mathrm{Te}_{3}$ и $\mathrm{Sb}_{2} \mathrm{Te}_{3}$ в составе твердого раствора и от температуры. Полученные результаты позволяют утверждать, что резкое уменьшение анизотропии магнитной восприимчивости при $T=293 \mathrm{~K}$ наблюдается в тех образцах, в которых, по данным оптических исследований, усиливается электрон-плазмонное взаимодействие, что обусловлено сближением энергий плазмона и межзонных переходов, формирующих край фундаментального поглощения.
\end{abstract}

DOI: 10.21883/FTP.2019.06.47726.35

\section{1. Введение}

Исследования кристаллов $\mathrm{Bi}_{2} \mathrm{Te}_{3}-\mathrm{Sb}_{2} \mathrm{Te}_{3}$ многочисленны и разнообразны, так как, с одной стороны, они являются высокоэффективными термоэлектрическими материалами [1], а с другой - вызывают интерес процессы, происходящие в электронной системе полупроводников с близкими значениями энергий элементарных возбуждений электронной и ионной систем. Изменением температуры, соотношением компонент, входящих в состав твердых растворов $\mathrm{Bi}_{2} \mathrm{Te}_{3}-\mathrm{Sb}_{2} \mathrm{Te}_{3}$, и их легированием примесями донорного и акцепторного типа можно добиться изменения интенсивности элементарных возбуждений, а также усиления их взаимодействия. $\mathrm{B}$ частности, кристаллы $\mathrm{Bi}_{2} \mathrm{Te}_{3}-\mathrm{Sb}_{2} \mathrm{Te}_{3}$ могут быть использованы для изучения взаимодействия одночастичных и коллективных возбуждений электронной системы, так называемого электрон-плазмонного взаимодействия, и его воздействия на физические характеристики полупроводникового материала, что является актуальной задачей физики конденсированного состояния.

\section{2. Кристаллы и образцы}

Исследовались монокристаллы твердых растворов системы $\mathrm{Bi}_{2} \mathrm{Te}_{3}-\mathrm{Sb}_{2} \mathrm{Te}_{3}$, содержащие 0, 10, 25, 40, 50, $60,65,70,80,90,99.5$ и 100 мол\% $\mathrm{Sb}_{2} \mathrm{Te}_{3}$. Кристаллы $\mathrm{Bi}_{2} \mathrm{Te}_{3}$ и $\mathrm{Sb}_{2} \mathrm{Te}_{3}$ обладают ромбоэдрической сингонией с пространственной группой типа $D_{3 d}^{5}$ по международной классификации [1]. Монокристаллы были выращены в Институте металлургии и материаловедения им. А.А. Байкова методом Чохральского [2].

В качестве исходных материалов использовались: Те, $\mathrm{Sb}, \mathrm{Bi}$, содержащие 99.9999 мас\% основного вещества.
Образцы для оптических и магнитных измерений вырезались из слитков при помощи электроискровой резки, с последующим удалением нарушенных при резке слоев толщиной до 100 мкм при помощи их травления в соляной кислоте.

\section{3. Методика и техника эксперимента}

Измерения спектров коэффициента отражения $R(v)$ проводились на фурье-спектрометрах PERKIN ELMER 1720X и SHIMADZU FTIR-8400S в диапазоне $400-4000 \mathrm{~cm}^{-1}$ с разрешением $1 \mathrm{~cm}^{-1}$. Регистрировались спектры отражения неполяризованного излучения от свежеприготовленного скола кристалла по плоскости, перпендикулярной тригональной оси кристалла $C_{3}$. Угол падения излучения на образец не превышал $8^{\circ}$. Измерения магнитной восприимчивости в диапазоне температур от 2 до $400 \mathrm{~K}$, с интервалом $3 \mathrm{~K}$, проводились в магнитных полях до 30 кЭ на сверхпроводящем квантовом интерферометре Джозефсона (SQUID-магнетометре) при двух ориентациях вектора напряженности магнитного поля $H$ по отношению к $C_{3} \quad\left(H \| C_{3} \quad\right.$ и $\left.H \perp C_{3}\right) \quad \chi_{\|}$и $\chi_{\perp}$ соответственно. Относительная погрешность измерений, выполненных на SQUID-магнетометре, не превышает $2 \%$.

\section{4. Анализ полученных результатов}

В ходе исследования магнитных свойств кристаллов $\mathrm{Bi}_{2} \mathrm{Te}_{3}-\mathrm{Sb}_{2} \mathrm{Te}_{3}$, в зависимости от состава и ориентации магнитного поля относительно кристаллографических осей, было обнаружено резкое уменьшение анизотропии магнитной восприимчивости в образцах, содержащих более 70 мол\% $\mathrm{Sb}_{2} \mathrm{Te}_{3}$, что показано на рис. 1 . 


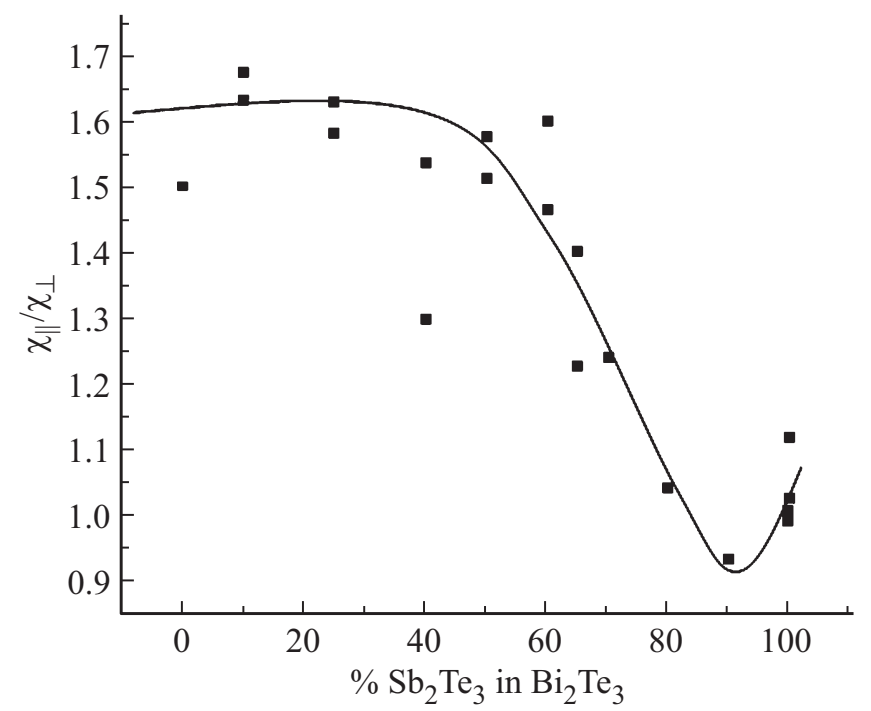

Рис. 1. Изменение величины анизотропии магнитной восприимчивости $\chi_{\|} / \chi_{\perp}$ в зависимости от соотношения компонент $\mathrm{Bi}_{2} \mathrm{Te}_{3}$ и $\mathrm{Sb}_{2} \mathrm{Te}_{3}$ в составе твердого раствора $\mathrm{Bi}_{2} \mathrm{Te}_{3}-\mathrm{Sb}_{2} \mathrm{Te}_{3}$. Данные получены при $T=293 \mathrm{~K}$.

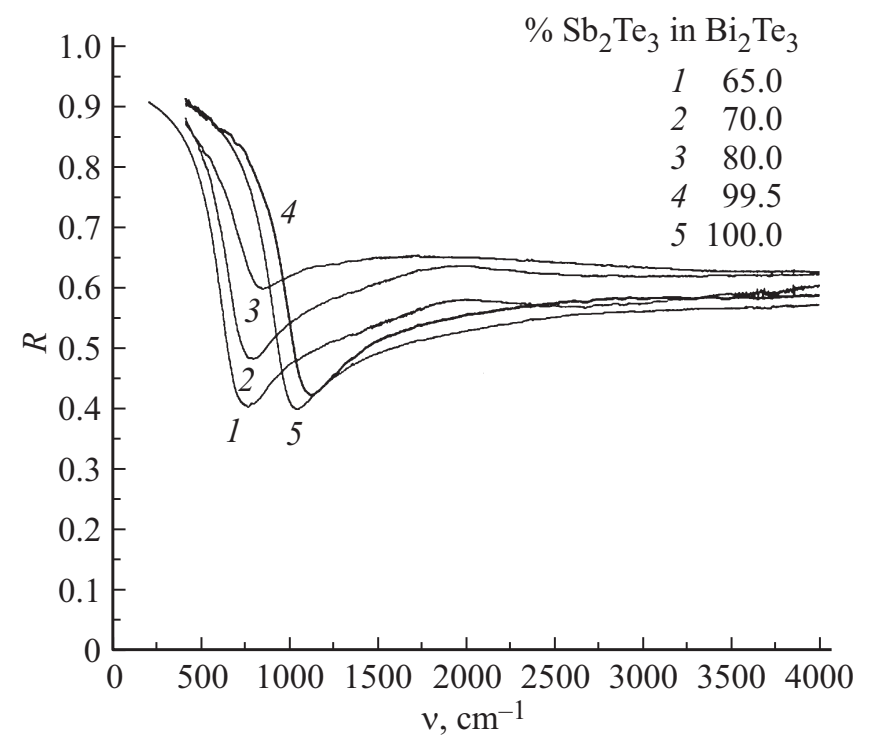

Рис. 2. Спектры отражения кристаллов твердых растворов $\mathrm{Bi}_{2} \mathrm{Te}_{3}-\mathrm{Sb}_{2} \mathrm{Te}_{3}$, полученные в неполяризованном излучении при $T=293 \mathrm{~K}$.

Также было установлено, что в области температур, соответствующих собственной проводимости, поведение магнитной восприимчивости кристаллов $\mathrm{Bi}_{2} \mathrm{Te}_{3}-\mathrm{Sb}_{2} \mathrm{Te}_{3}$ удается описать в рамках подходов Паули и ЛандауПайерлса, если учитывать не только хаотизацию направлений магнитных моментов, но и изменение таких параметров электронной системы кристалла, как концентрация свободных носителей и их эффективная масса [3]. Анализ результатов оптических исследований, а именно спектров отражения в инфракрасной области спектра, структура которых обусловлена взаимодействием плаз- мы свободных носителей заряда с электромагнитным излучением, показал, что в кристаллах, в которых наблюдается переход в изотропное магнитное состояние, обнаруживаются особенности, связанные с деформацией плазменного края. Так, на рис. 2, на котором представлены спектры плазменного отражения ряда кристаллов $\mathrm{Bi}_{2} \mathrm{Te}_{3}-\mathrm{Sb}_{2} \mathrm{Te}_{3}$, полученные при $T=293 \mathrm{~K}$, наблюдается смещение плазменного минимума в высокочастотный диапазон по мере увеличения процентного содержания $\mathrm{Sb}_{2} \mathrm{Te}_{3}$ в составе твердого раствора. При этом в спектрах отражения кристаллов, содержащих от 50 до 80 мол\% $\mathrm{Sb}_{2} \mathrm{Te}_{3}$, проявляются особенности, а также наблюдается усиление затухания плазменных колебаний, фиксирующееся по уменьшению оптических времен релаксации, приведенных в таблице. Указанные особенности связаны c деформацией плазменного края, заключающейся в уменьшении наклона кривой спектра отражения и увеличении коэффициента отражения в минимуме, что также отражено на рис. 2. Максимальная деформация плазменного края наблюдается при содержании 80 мол\% $\mathrm{Sb}_{2} \mathrm{Te}_{3}$ в $\mathrm{Bi}_{2} \mathrm{Te}_{3}-\mathrm{Sb}_{2} \mathrm{Te}_{3}$. Установлено, что величина деформаций нарастает по мере сближения энергий межзонных переходов и плазмонов, представляющих резонансные возбуждения одночастичных и коллективных колебаний электронной системы, что приводит к усилению электрон-плазмонного взаимодействия [4]. Действительно, обычно в полупроводниках в высокочастотной, по отношению к плазменному краю, области спектра существует окно относительной прозрачности материала, которое заканчивается на частотах, характерных для начала межзонных переходов, формирующих край фундаментального поглощения. В случае сближения плазменного края и края фундаментального поглощения диапазон частот, на которых наблюдается относительно высокое пропускание излучения, уменьшается, а на спектрах

Параметры плазменных колебаний свободных носителей заряда кристаллов твердых растворов $\mathrm{Bi}_{2} \mathrm{Te}_{3}-\mathrm{Sb}_{2} \mathrm{Te}_{3}$, полученные при $T=293 \mathrm{~K}$

\begin{tabular}{c|c|c|c|c|c}
\hline № п.п. & Состав & $\%$ & $\begin{array}{c}\omega_{p} \cdot 10^{13}, \\
\mathrm{c}^{-1}\end{array}$ & $\varepsilon_{\infty}$ & $\begin{array}{c}\tau_{\text {орt }} \cdot 10^{-14} \\
\mathrm{c}\end{array}$ \\
\hline 1 & $\mathrm{Bi}_{2} \mathrm{Te}_{3}$ & 0 & 3.3 & 45 & 2.5 \\
2 & $\mathrm{Bi}_{1.8} \mathrm{Sb}_{0.2} \mathrm{Te}_{3}$ & 10 & 4.7 & 41 & 2.5 \\
3 & $\mathrm{Bi}_{1.8} \mathrm{Sb}_{0.2} \mathrm{Te}_{3}$ & 10 & 3.5 & 52 & 3.1 \\
4 & $\mathrm{Bi}_{1.2} \mathrm{Sb}_{0.8} \mathrm{Te}_{3}$ & 40 & 8.3 & 57 & 2.6 \\
5 & $\mathrm{BiSbTe}_{3}$ & 50 & 7.8 & 78 & 2.2 \\
6 & $\mathrm{Bi}_{0.8} \mathrm{Sb}_{1.2} \mathrm{Te}_{3}$ & 60 & 10.4 & 81 & 2.4 \\
7 & $\mathrm{Bi}_{0.7} \mathrm{Sb}_{1.3} \mathrm{Te}_{3}$ & 65 & 12.6 & 85 & 2.3 \\
8 & $\mathrm{Bi}_{0.6} \mathrm{Sb}_{1.4} \mathrm{Te}_{3}$ & 70 & 12.9 & 80 & 1.7 \\
9 & $\mathrm{Bi}_{0.6} \mathrm{Sb}_{1.4} \mathrm{Te}_{3}$ & 70 & 13.0 & 81 & 1.8 \\
10 & $\mathrm{Bi}_{0.4} \mathrm{Sb}_{1.6} \mathrm{Te}_{3}$ & 80 & 14.2 & 89 & 1.63 \\
11 & $\mathrm{Bi}_{0.2} \mathrm{Sb}_{1.8} \mathrm{Te}_{3}$ & 90 & 14.1 & 83 & 1.54 \\
12 & $\mathrm{Bi}_{0.01} \mathrm{Sb}_{1.99} \mathrm{Te}_{3}$ & 99.5 & 19.4 & 62 & 1.66 \\
13 & $\mathrm{Bi}_{0.01} \mathrm{Sb}_{1.99} \mathrm{Te}_{3}$ & 99.5 & 19.4 & 69 & 2.06 \\
14 & $\mathrm{Sb}_{2} \mathrm{Te}_{3}$ & 100 & 17.9 & 55 & 2.15 \\
15 & $\mathrm{Sb}_{2} \mathrm{Te}_{3}$ & 100 & 17.5 & 57 & 2.93
\end{tabular}


отражения наблюдается деформация плазменного края. Во многом это обусловлено тем, что вблизи плазменной частоты действительная часть функции диэлектрической проницаемости оказывается близка к нулю, что и обеспечивает возможность проявления особенностей, связанных с межзонными переходами, обычно слабо выделяющимися на фоне поляризационных процессов, обусловленных свободными носителями заряда. В связи с этим с высокочастотной стороны от плазменного края, где действительная часть функции диэлектрической проницаемости положительна и мала по абсолютной величине, даже слабое влияние межзонного перехода вызывает заметное изменение поведения оптических функций. Однако, как только плазменная частота становится больше частоты межзонного перехода, интенсивность последнего резко уменьшается вследствие экранировки падающего на кристалл электромагнитного излучения колебаниями свободных носителей заряда [5]. Именно это и наблюдается в случае увеличения содержания $\mathrm{Sb}_{2} \mathrm{Te}_{3}$ в $\mathrm{Bi}_{2} \mathrm{Te}_{3}-\mathrm{Sb}_{2} \mathrm{Te}_{3}$ до 80 мол\%. Так, в кристаллах, содержащих более 90 мол\% $\mathrm{Sb}_{2} \mathrm{Te}_{3}$, плазменная частота быстро увеличивается, достигая значений $\sim 1000 \mathrm{~cm}^{-1}$. При этом особенности, характерные для межзонных переходов, исчезают, и спектры отражения хорошо описываются в рамках классической теории взаимодействия электромагнитного излучения с плазмой свободных носителей заряда.

\section{5. Заключение}

Таким образом, электрон-плазмонное взаимодействие, обнаруживающееся в ходе оптического эксперимента по деформации спектров отражения, наиболее вероятно является и причиной уменьшения анизотропии магнитной восприимчивости. Так как величина магнитной восприимчивости формируется всем объемом образца, находящегося в магнитном поле, а не только его поверхностью, как в оптическом эксперименте, связанном с исследованием коэффициента отражения, то можно утверждать, что электрон-плазмонное взаимодействие влияет на состояние электронной системы кристалла в целом. В связи с этим при заданном соотношении компонент $\mathrm{Bi}_{2} \mathrm{Te}_{3}$ и $\mathrm{Sb}_{2} \mathrm{Te}_{3}$ в составе твердого раствора и температуры будет существовать определенная интенсивность электрон-плазмонного взаимодействия, которую необходимо учитывать при анализе закономерностей в поведении физических характеристик, формирующихся в объеме кристалла и зависящих от параметров электронной системы. Всестороннее исследование этого явления, наблюдающегося в материалах, использующихся для создания термоэлектрических преобразователей энергии, представляет интерес как с практической, так и с фундаментальной точек зрения.

\section{Список литературы}

[1] Б.М. Гольцман, В.А. Кудинов, И.А. Смирнов. Полупроводниковые термоэлектрические материалы на основе $\mathrm{Bi}_{2} \mathrm{Te}_{3}$ (М., Наука, 1972).

[2] Л.Д. Иванова, Ю.В. Гранаткина. Изв. АН СССР. Неорг. матер., 36 (7), 810 (2000).

[3] Н.П. Степанов, В.Ю. Наливкин. Неорг. матер., 48 (3), 297 (2015).

[4] Д. Пайнс. УФН, 62 (4), 399 (1957).

[5] Н.П. Степанов, А.А. Калашников, Ю.В. Улашкевич. Опт. и спектр., 117 (3), 415 (2014).

Редактор А.Н. Смирнов

\section{Optical and Magnetic Correlation crystals $\mathbf{B i}_{2} \mathbf{T e}_{3}-\mathbf{S b}_{2} \mathbf{T e}_{3}$}

N.P. Stepanov ${ }^{1}$, A.K. Gilfanov ${ }^{2}$, E.N. Trubitsyna ${ }^{2}$

${ }^{1}$ Zabaikalsky State University, 672039 Chita, Russia

${ }^{2}$ Zabaikalsky Institute of Entrepreneurship, 672039 Chita, Russia

Abstract Magnetic and optical properties of $\mathrm{Bi}_{2} \mathrm{Te}_{3}-\mathrm{Sb}_{2} \mathrm{Te}_{3}$ alloy crystals were studied depending on the composition of the solid solution and on temperature. Sharp decrease in the anisotropy of magnetic susceptibility at $T=293 \mathrm{~K}$ is observed in those samples in which, according to optical studies, electronplasmon interaction is intensified. The increase of the electronplasmon interaction is caused by the convergence of plasmon energies and interband transitions that form the fundamental absorption edge. 